\title{
Building 3D anatomical scenes on the Web
}

\author{
F. Evesque, S. Gerlach, R. D. Hersch \\ Ecole Polytechnique Fédérale de Lausanne (EPFL) \\ http://visiblehuman.epfl.ch
}

\begin{abstract}
We propose a new service for building user-defined 3D anatomic structures on the Web. The Web server is connected to a data base storing more than 1000 3D anatomic models reconstructed from the Visible Human. Users may combine existing models as well as planar oblique slices in order to create their own structured anatomic scenes. Furthermore, they may record sequences of scene construction and visualization actions. These actions enable the server to construct high-quality video animations, downloadable by the user. Professionals and students in anatomy, medicine and related disciplines are invited to use the server and create their own anatomic scenes.
\end{abstract}

\section{Keywords}

Visible Human, anatomy, anatomic models, interactive construction of 3D scenes, volume visualization, surface reconstruction, applet-based rendering engine, Java.

\section{Introduction}

Since its creation in June 1998, EPFL's Visible Human Web Server (http://visiblehuman.epfl.ch) aims at offering a comprehensive set of services for exploring the human anatomy in all its dimensions. The services rely on the Visible Human datasets licensed by the National Library of Medicine [1]. Web users may freely extract arbitrarily oriented and positioned slices [2], curved surfaces, slice animations [3], or navigate in real-time within the volumic data set [4].

Since August 2001, we offer on the Web a new virtual construction kit allowing users to construct 3D anatomical scenes by combining planar slices and 3D anatomic structures.

We have reconstructed the surface models of 3D anatomic structures from the Classified and Segmented Visible Human Male volume data set provided by Gold Standard Multimedia [5].

To create this new service, we had to meet the following challenges:

- create compact surface models from the segmented and classified 3D volume of the Visible Human;

- develop a 3D rendering engine allowing Web clients to visualize these anatomic surface models in real-time;

- combine the models with textured slices extracted by the Visible Human Slice Server;

- offer a user interface for interactively creating sophisticated $3 \mathrm{D}$ anatomical scenes.

We created a Java 1.1 applet with a custom 3D rendering engine and a user interface capable of interacting with a database (Fig. 1). The database resides on the server and contains the description of anatomic surface models and scenes. It also describes user-defined
3D scene animations. Animated 3D scene descriptions are used for the synthesis of high-quality video animations. Users may construct their own 3D scenes and animations and share them with other users.

In section 2, we show how the 3D surface models were reconstructed. In section 3, we describe the adopted 3D rendering technique, which supports both opaque and transparent models. In section 4 , we give a description of the user interface and its functionality. The conclusions are drawn in section 5 .

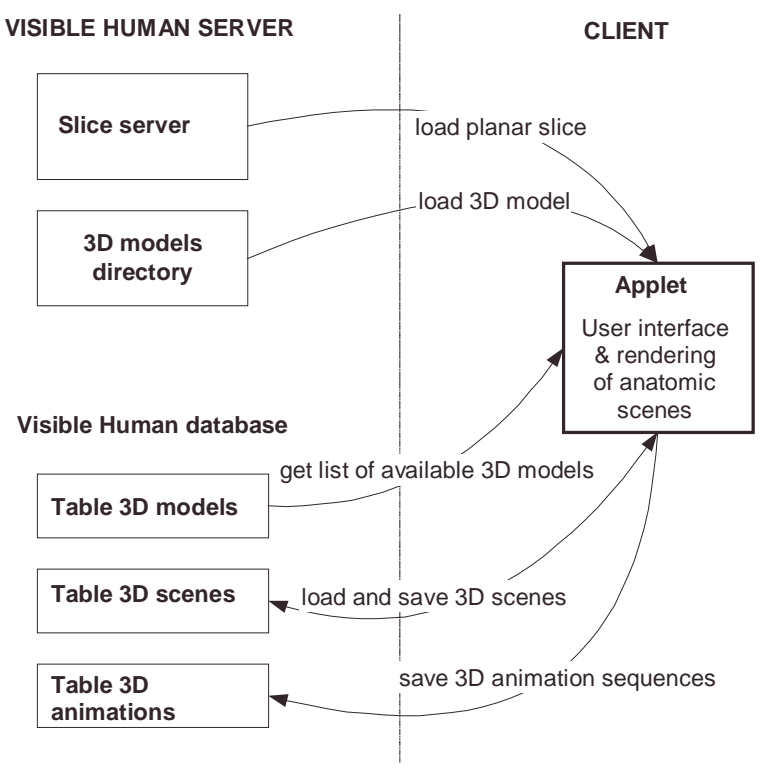

Fig. 1. Global architecture of the 3D anatomic scene server

\section{Related work}

Several related projects aim at making 3D anatomy accessible to students and professionals as well as to a larger public. The VoxelMan system distributed on CD-ROM by Springer Verlag allows users to interact and explore anatomy structures via precomputed "Intelligent QuickTime VR Movies" [6]. The Brain Browser, a system for visualizing the brain (http://sig.biostr.washington.edu/projects/brain/demos.html) relies on the server side computation of 3D brain structure images and their visualization in the client's applet [7]. In the Anatomy Browser (http://splweb.bwh.harvard.edu:8000/pages/papers/ AnatomyBrowser/current/index.html), 3D projections along the main orientations as well as axial, coronal and sagittal slices, including labelling information is made available to the client applet [8]. In another project, a VRML interface is used as a tool for visualizing a combination of $3 \mathrm{D}$ structures reconstructed from 
MRI images and planar slices [9]. In our project, the client applet provides extensive interaction capabilities for constructing anatomic scenes, is capable of displaying 3D structures in real time, offers both transparent and opaque display modes and allows to record user interactions in order to produce high-quality video sequences.

\section{Reconstruction of 3D models}

The Visible Human Male dataset [1] licensed by the National Library of Medicine has been "segmented and classified" by Gold Standard Multimedia [5]. This segmented dataset is a collection of 1878 slices with resolution $1760 \times 1024$ pixels. It forms a volume of $587 \times 341 \times 1878 \mathrm{~mm}$, with each voxel representing $0.33 \times 0.33 \times 1 \mathrm{~mm}$ and containing an index to the corresponding anatomical structure.

An index file describes for each index the anatomical structure it represents, its body region, and its anatomic system. A total of 1400 anatomic names are classified.

In the present section, we describe the creation of 3D surface models from the segmented volume dataset. Anatomic structures should be represented as compact surface descriptions, downloadable by an applet.

The Marching Cubes algorithm [10] is an efficient method to compute the surface contouring a particular volume. This algorithm has already been successfully applied to the Visible Human CT data [13].

Sets of voxels with the same index form a volume whose surface is to reconstructed. The Marching Cubes algorithm generates a triangle mesh with a large number of triangles. By applying the decimation method [11] provided by the Visualization Toolkit (VTK [12]), the number of triangles can be considerably reduced.

Triangles are stored as triangle strips, a compact representation in which triangles are connected edge to edge. Models formed of triangle strips are saved as compressed binary files.

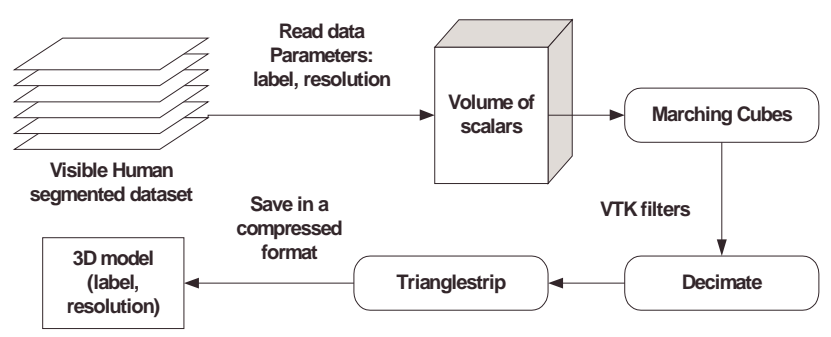

Fig. 2. Reconstruction of surface models

The Marching Cubes algorithm has been applied to the segmented Visible Human dataset at five different resolutions, with marching cube voxel elements comprising between 2 pts x 2 pts x 1 pts of the original dataset (real size: $0.66 \mathrm{~mm} \times 0.66 \mathrm{~mm} \times 1 \mathrm{~mm}$ ) and 32 pts $\mathrm{x} 32$ pts $\mathrm{x} 16$ pts of the original dataset (real size: $10.66 \mathrm{~mm} \times$ $10.66 \mathrm{~mm} \times 16 \mathrm{~mm})$.

We applied the decimation filter of VTK to each model and at each resolution in order to reduce the number of triangles generated by the Marching Cubes algorithm. In average, the decimated models allowed to reduce the initial number of triangles by a factor of five.
For each anatomical structure (e.g. the vertebra in Fig. 3), we created 3D surface models at different resolutions. The number of triangles varies between 100 and 50000 triangles, corresponding to $3 \mathrm{D}$ model files of $1 \mathrm{~KB}$ to $250 \mathrm{~KB}$. The resulting size depends on the resolution, the complexity of the shape and the precision of the segmentation.

The quality of each 3D model at each resolution was evaluated by anatomists so as to retain only models of interest. In order to provide sufficient flexibility, we tried to keep three levels of resolution, high, middle and low (Fig. 3). Levels of resolution providing too much noise and too many unimportant details as well as levels of resolution too coarse for representing a given anatomic structure were discarded.

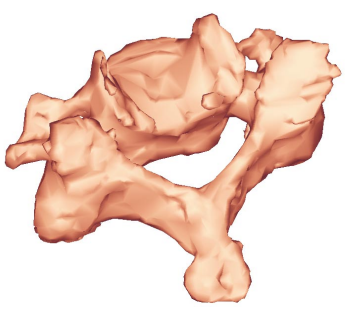

(a) high-resolution vertebra, 6676 triangles

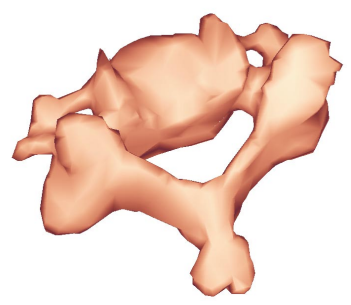

(b) middle-resolution vertebra 1784 triangles
Fig. 3. Model of the C-4 vertebra at high and middle resolution

\section{Rendering}

The Web service we offer allows to combine the visualization of 3D anatomic surfaces and planar slices. In addition, its user interface must allow to carry out complex operations, such as the translation and rotation of a planar slice within a 3D anatomic scene. Furthermore, it should allow to search for anatomic models and to add them to an existing scene.

Due to the fact that most browsers only support Java 1.1 and that an elaborate user interface was needed, we decided neither to user Java3D (an extension of Java 2) nor to make use of a VRML browser. We created within a Java 1.1 applet a custom 3D rendering engine. This applet comprises both a visualization interface and a sophisticated user interface for selecting models and building model hierarchies. This solution does not require users to download a plug-in.

The rendering engine is capable of displaying in real-time a 3D scene containing more than 100'000 triangles. The following features were adopted: Gouraud shading, texture mapping for displaying the slices extracted by the slice server, transparency in order to display several anatomic structures hiding one another and identification of the displayed 3D models in order to provide support for interactive model selection.

Since the 3D models are formed by triangles, the 3D engine only accepts triangles as surface primitives. For simplification purposes, only one light source at the same position as the camera is available. For hidden surface removal, the well-known Z-buffer algorithm is applied.

In addition to the Z- and color buffers, we use a third buffer incorporating the anatomic structure model identifier. Anatomic models can therefore be easily selected and highlighted by a mouse cursor. 
For the transparent display of anatomic structures, we use a scanline buffer (s-buffer) algorithm. This algorithm allows the correct rendering of complex anatomic scenes comprising overlapping semi-transparent models and/or slices. For each display scanline, we establish a list of horizontal segments representing the intersection of the scanline with the projected triangles. We memorize the coordinates of the horizontal segment vertices as well as their depth, intensity and possibly texture coordinates.

The list of horizontal segments associated to a scanline contains the necessary information in order to obtain for each pixel the segments contributing to its color. These segments are sorted by depth and the pixel's final color is obtained by alpha blending.

We tested the rendering results on different scenes. The tests were realized on a Pentium III $500 \mathrm{MHz}$, with Internet Explorer 5 and the latest Microsoft virtual machine for Java (version 5.0.0.3802).

The scene is displayed in a window of $420 \times 360$ pixels. In opacity mode, since all 3D models are closed, back faces are never visible and do not need to be rendered. In the case of transparent rendering, triangles belonging to the back face also contribute to the displayed scene.

The results show that the scan-line buffer algorithm for transparent rendering is not fast enough for real-time animation. For opaque rendering however, the Z-buffer algorithm yields acceptable frame rates even for very complex scenes.

Therefore, in order to preserve interactivity, we decided to display the scene with all models opaque during camera movement. Once the camera movement has come to an end, the final scene may be rendered in transparent mode.

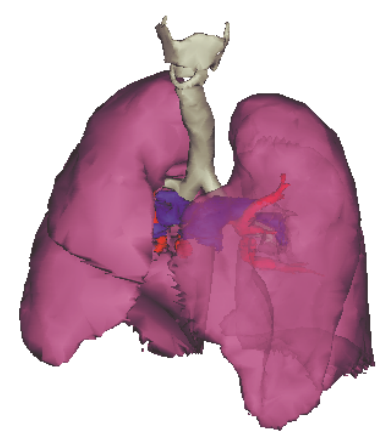

Respiratory system:

Number of triangles: 35000

\begin{tabular}{|l|l|}
\hline Mode & Frame rate \\
\hline Opaque & $6.2 \mathrm{fps}$ \\
\hline Transparency & $0.4 \mathrm{fps}$ \\
\hline
\end{tabular}

fps=number of frames per second.

Digestive system with 2 textured slices:

Number of triangles: 72000

\begin{tabular}{|l|l|}
\hline Mode & Frame rate \\
\hline Opaque & $3.4 \mathrm{fps}$ \\
\hline Transparency & $0.25 \mathrm{fps}$ \\
\hline
\end{tabular}

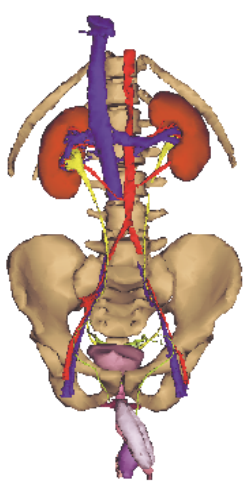

Urogenital system:

Number of triangles: 145000

\begin{tabular}{|l|l|}
\hline Mode & Frame rate \\
\hline Opaque & $2.6 \mathrm{fps}$ \\
\hline Transparency & $0.3 \mathrm{fps}$ \\
\hline
\end{tabular}

Fig. 4. Rendered anatomic scenes

\section{User Interface}

The environment for the creation of 3D scenes and their visualization requires the following functionality:

- Loading and displaying 3D models and planar slices

- Interactive navigation: rotation, zoom and translation

- Slice movements: rotation and translation in 3D space

- Edition of the model parameters: visibility, color, opacity, and name

- Edition of the anatomic scene structure represented by a tree with selectable and dragable directories and items (Fig. 6)

- Loading and saving 3D scenes in the database

- Recording sequences of navigation actions in order to produce off-line high quality video animations.

Fig. 5 shows the applet's user interface. It is divided into four parts. On the top, a toolbar contains buttons for loading and saving 3D scenes (new, open, and save) and buttons for changing the mouse modes (camera rotation, zoom, translation, selection of anatomic structures, slice extraction and slice movements, animation recording). On the right part of the applet, the current view of the scene is displayed. Below, a text field shows the name of the anatomic structure currently selected by the mouse. On the left side, a panel made of the 3 tabs View, Edit and Search and load allows users to interact with the current scene.

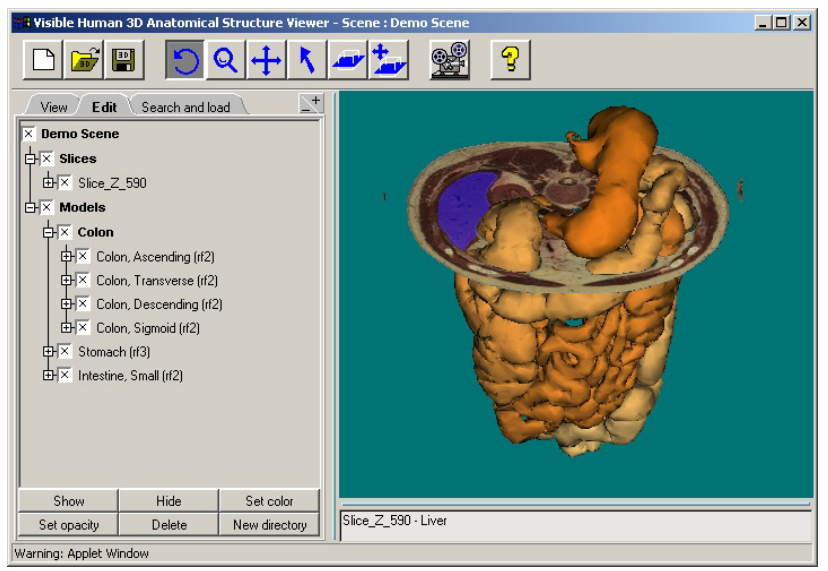

Fig. 5. Screenshot of the applet 


\subsection{Loading 3D models and labeled slices}

Users may load a specific anatomic model by querying in the Search and load tab the Visible Human database. They may type a name of an anatomic structure and, in addition, specify a particular anatomic system or body region.

The query may yield several anatomic models or structures composed of several models. The user may then select the substructures or models he would like to load and display.

One may load a planar slice by switching to the Slice extraction mouse mode and by clicking on a specific surface position of a 3D model. A small pop-up window allows to specify the desired slice orientation, resolution and size. The selected slice will intersect the model at the specified position.

Once a slice is loaded, further 3D models may be easily added to the scene by clicking, in Selection mouse mode, on a specific slice location. The corresponding $2 \mathrm{D}$ anatomic substructure is highlighted and its name is given at the bottom of the window. The system will then automatically, in the Search and load tab, propose the corresponding 3D anatomic structure for subsequent loading.

\subsection{Interactive navigation}

In order to modify the point of view of the scene, i.e. the camera position, three mouse modes are available, which correspond to rotation, zoom and translation. The user may select these mouse modes in the toolbar or with different mouse buttons.

A rotation is carried out around the horizontal and vertical axes by pressing with the mouse cursor on one of the display quadrants (rotation around vertical axis: $-45^{\circ} . .45^{\circ}, 135^{\circ} . .225^{\circ}$, rotation around horizontal axis: $\left.45^{\circ} . .135^{\circ}, 225^{\circ} . .315^{\circ}\right)$. By keeping the mouse pressed, a continuous rotation is obtained. The farther the mouse from the center of the display, the quicker the scene rotates. Zoom and translation are obtained by mouse "click and drag" actions.

\subsection{Slice translations and rotations}

Slices extracted along a main orientation (axial, sagittal or coronal) may also be translated and rotated. The user may translate a slice either along its normal or within its plane, and rotate it around the main slice axes.

The slice is moved by clicking and dragging it (translation: simple click \& drag, rotation: shift-click). During the slice movement, the slice is displayed without texture. Once the mouse button is released, a new texture is loaded. This allows to freely position and orient a planar slice within a 3D scene.

\subsection{Editing of the parameters}

In the "Edit" tab, the parameters of the models such as their visibility, their color, their opacity or their name may be modified. Changes can be done individually or applied at once to a list of selected models. Selection of the models is done by clicking either on the name of the model or on the corresponding anatomic structure model on the screen. For multiple selection, the "Ctrl" and "Shift" keys can be used.

\subsection{Scene structure editing}

The scene is organized as a tree of directories (Fig. 6). Leaf nodes are individual anatomic models or slices. In the Edit tab, newly loaded anatomic models appear in the directory "Models" and planar slices in the directory "Slices". However, the user may define his own structure: he may create other directories. By "drag and drop", he may move models or respectively directories from one place to another.

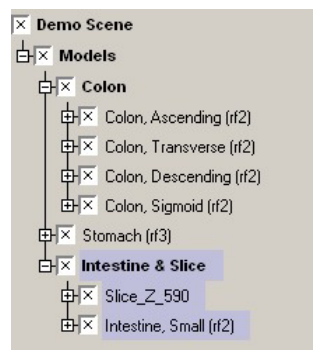

Fig. 6. Creation and editing of a directory

Grouping anatomic structures in directories allows to easily modify their display properties (visibility, color, opacity). For instance, one may visualize or hide in the display window the models contained in a directory by just clicking on the directory's check-box.

\subsection{Loading and saving anatomic scenes}

When a user a scene, both the parameters of the scene (background color, camera position, scene structure) and the parameters of each anatomic model (color, visibility, name, opacity) are saved. These parameters are saved under the user's personal entry in the Visible Human server database (Fig. 7).

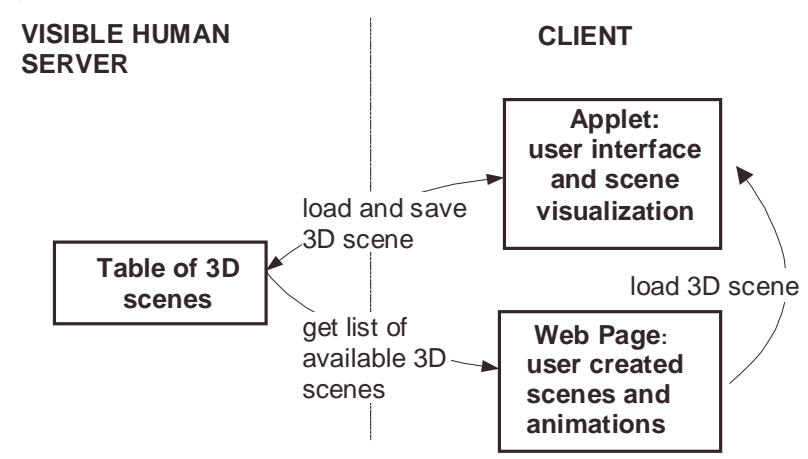

Fig. 7. Interaction between the applet and the server for loading and saving $3 \mathrm{D}$ anatomic scenes

On the Web page containing the user's personal data (accessible by clicking on the item "saved markers, 3D scenes and 3D animations" of the main server page), the list of the available scenes is displayed. A thumbnail enables to quickly recognize a scene. The user may load a personal scene, a scene shared with him by another user, or a public scene (Fig. 8).

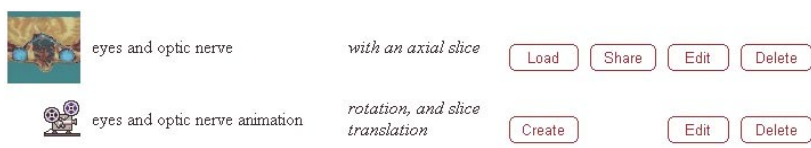

Fig. 8. Web page allowing to load 3D scenes or to create video animations

5.7 Synthesis and recording of high quality animations

A user may record a sequence of actions in order to produce offline high quality animations. The database stores the succession of 
navigation and visualization actions such as scene rotation, zoom, and translation, slice translation, and modification of the visibility of anatomic model parameters.

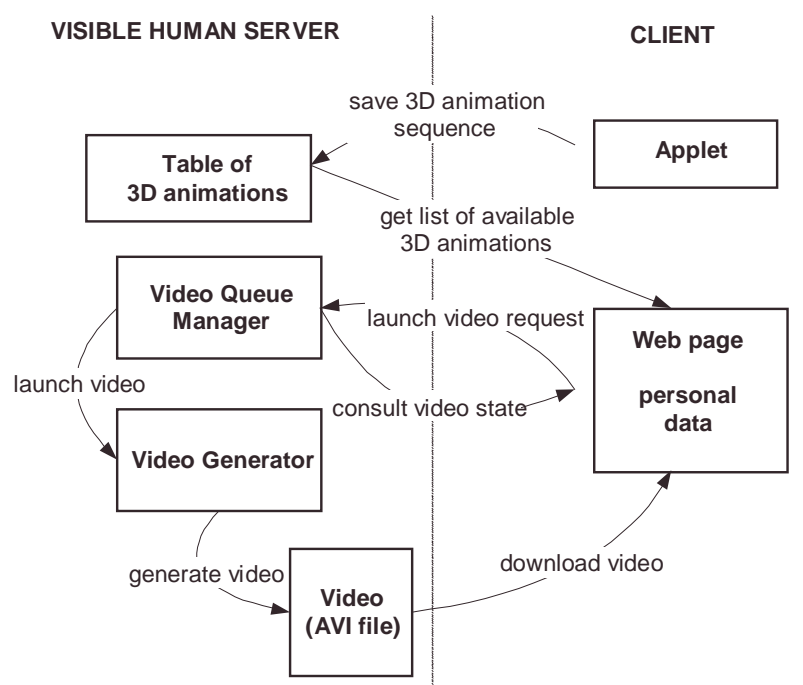

Fig. 9. Synthesis of a video animation

In order to produce a video animation, the user defines on the Web page referencing his scene animation the parameters of the desired video, i.e. its dimensions and frame rate. He then asks the server to produce the video. The request is queued and forwarded to the Video Generator (Fig. 9). The Video Generator synthesizes as many frames as required, according to the desired dimensions and frame rate and converts them into a video in AVI format.

Since it is processed off-line by the server, the quality of the synthesized video is higher than the animation displayed in the applet. The frame rate is constant, camera movements are smooth, and other advanced features such as textured slice translation, and animations with partly transparent anatomic structures are supported.

After synthesis of the video, a link is available on the Web page allowing to download the video. This link remains valid during the next 24 hours. Afterwards, the video is automatically removed from the server.

Sample animations may be downloaded from the Visible Human Web server (http://visiblehuman.epfl.ch/samples.php).

\section{Conclusions}

EPFL's Visible Human Web Server allows users with a Javaenabled Web browser to easily construct and visualize 3D anatomical scenes. In addition, users may produce high quality animations incorporating interesting 3D model animations and visibility modifications.

The 3D surface models representing anatomic structures can be freely combined with extracted planar slices. Anatomic scenes of high value can be created which bridge the gap between planar slices and 3D representations.

This service opens new perspectives for teaching anatomy to paramedical and medical professions. The service is free. Once registered, users may, under their personal entry, create, edit and structure anatomic scenes. They may share them with other users.
We invite the readers to test this new and unique service on the Web (http://visiblehuman.epfl.ch). Professionals and students active in anatomy and medicine are invited to create their own anatomic scenes and possibly share them with their colleagues and with the public.

\section{Acknowledgements}

The authors would like to thank Prof. J. P. Hornung and his student Tri-Linh Lu, Medical Faculty of the University of Lausanne, for creating hierarchies of 3D anatomical structures and evaluating the quality of the reconstructed 3D models. We also thank Dr Nadia Danon for having created the anatomic scene shown in the appendix.

\section{References}

[1] M. Ackerman, The Visible Human project, Proceedings of the IEEE, Vol. 86, No. 3, March 1998, pp 504-511

[2] R.D. Hersch et al., "The Visible Human Slice Web Server: A first Assessment", Symposium on Electronic Imaging 2000, Conference on Internet Imaging, San Jose, Ca, Jan. 2000, SPIE Vol. 3964, 253-258.

[3] J.C. Bessaud, R.D. Hersch, "The Visible Human Slice Sequence Animation Web Server", IS\&T/SPIE Conference on Internet Imaging II, Jan. 25-31, San Jose, 2001, SPIE Vol. 4311, 341-348.

[4] S. Gerlach, R.D. Hersch, A Web-based Real-time Visible Human Navigator, IEEE Internet Computing, accepted for publication

[5] GSM, Labeled Visible Human dataset, http://www.gsm.com/ docs/products/segclass.htm

[6] R. Schubert, B. Pflesser, A. Pommert, K. Priesmeyer, M. Riemer, T. Schiemann, U. Tiede, P. Steiner, and K. H. Höhne, "Interactive volume visualization using "intelligent movies"," in Medicine meets Virtual Reality, Proc. MMVR '99 (J. D. Westwood, et. al. eds.), vol. 62 of Health Technology and Informatics, Amsterdam: IOS Press, 1999, 321-327,

[7] A. Poliakov, E. Albright, D. Corina, G. Ojemann, R. F. Martin and J. F. Brinkley, Server-based Approach to Web Visualization of Integrated 3-D Medical Image Data, Proc. AMIA'2001,

http://www1.biostr.washington.edu/ andrew/AMIA_2001_fi nal.html

[8] P. Golland, R. Kikinis, M. Halle, C. Umans, W.E.L. Grimson, M.E. Shenton, J.A. Richolt. "AnatomyBrowser: A Novel Approach to Visualization and Integration of Medical Information", Journal of Computer Assisted Surgery, Vol. 4, 1999, 129-143

[9] P.A Warrick, W.R.J. Funnell, A VRML-based anatomical visualization tool for medical education. IEEE Trans. Inf. Technol. Biomed. Vol. 2, No. 2, 1998, 55-61

[10] W.E. Lorensen, H.E. Cline, "Marching Cubes: a high resolution 3D surface construction algorithm", Proc. SIGGRAPH'87, Computer Graphics, Vol. 21, No. 3, 1987, 163-169

[11] W.J. Shroeder, J.A. Zarge, W.E. Lorensen, "Decimation of Triangle Meshes", Proc. SIGGRAPH'92, Computer Graphics, Vol. 26, No 2, July 1992, 65-70 
[12] W. Shroeder, K. Martin, B. Lorensen, "The Visualisation Toolkit", http://www.kitware.com/vtk

[13] W.E. Lorensen, "Marching Through the Visible Human", The Visible Human Project Conference Proceedings, National Institutes of Health, Bethesda, October 7 \& 8, 1996, http://www.nlm.nih.gov/research/visible/vhp_conf/vhpconf.h $\underline{\mathrm{tm}}$ 


\section{Appendix}

The picture below shows a slightly oblique back view of the abdomen comprising the spine (rose), the kidneys (lilac), the liver (light purple), the stomach (red) and the colon (yellow-brown). The lungs are shown in transparent blue and reveal the heart ventricles (on the coronal slice).

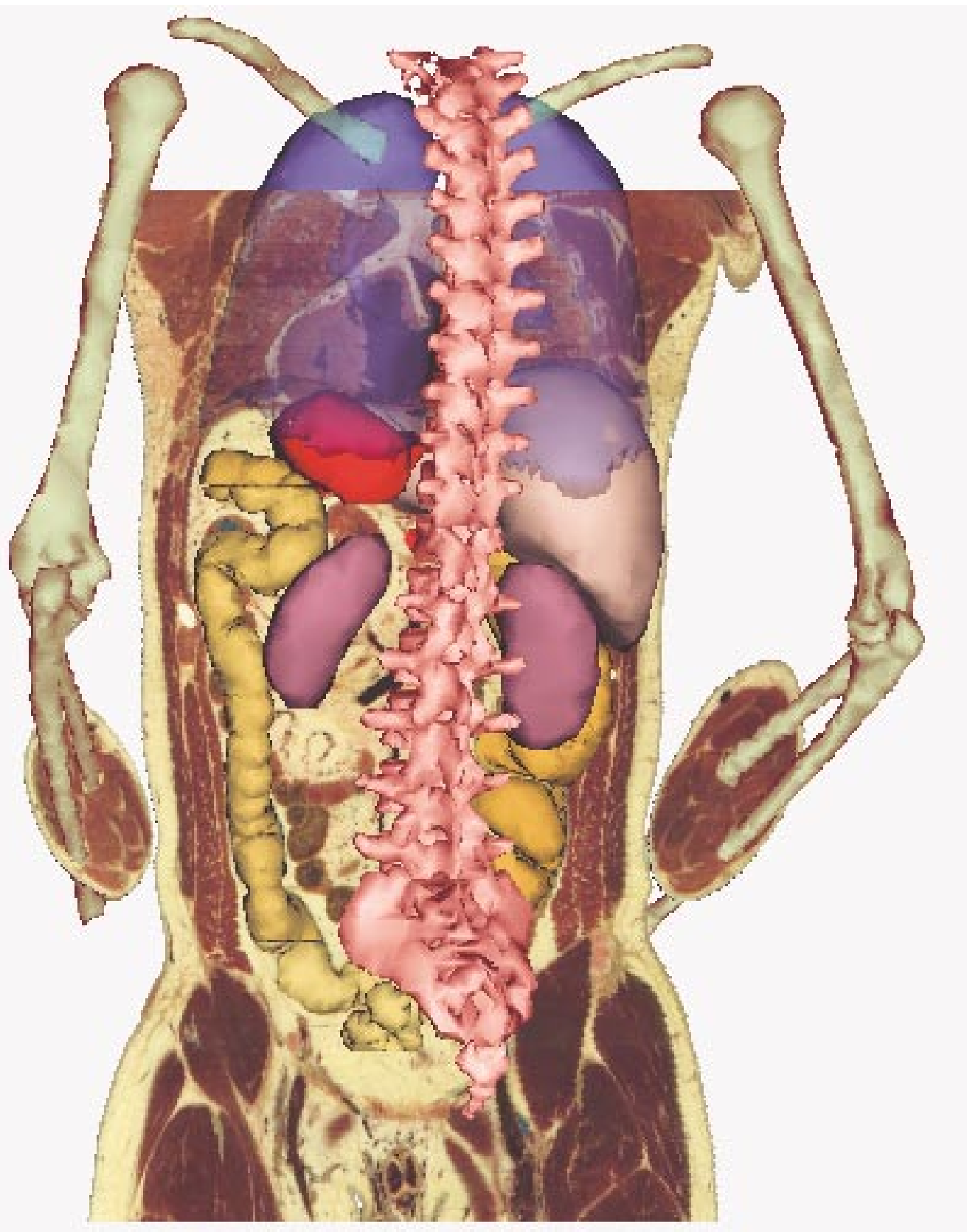

